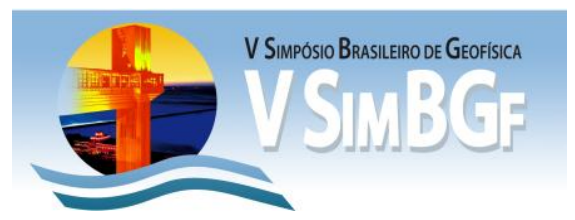

\title{
Integração de Dados Geofísicos e Utilização de Técnicas de Filtragem da Anomalia Magnética de Intensidade Total ao Sudoeste da Folha Minas Camaquã - RS.
}

Luis Felipe de Melo Tassinari', Universidade Federal do Pampa; Cauê Vivian Hess, Universidade Federal do Pampa; Guilherme Freitas Ilha, Universidade Federal do Pampa; Marcos Vinícios da S. Ferreira, Universidade Federal do Pampa; Mario J. Tomas Rosales, Universidade Federal do Pampa

Copyright 2012, SBGf - Sociedade Brasileira de Geofísica

Este texto foi preparado para a apresentação no V Simpósio Brasileiro de Geofísica, Salvador, 27 a 29 de novembro de 2012. Seu conteúdo foi revisado pelo Comitê Técnico do V SimBGf, mas não necessariamente representa a opinião da SBGf ou de seus associados. É proibida a reprodução total ou parcial deste material para propósitos comerciais sem prévia autorização da SBGf.

\section{Resumo}

Esse trabalho utiliza como metodologia principal a integração de dados aeromagnéticos em suas transformações de seu campo potencial, dados de levantamentos terrestres de susceptibilidade magnética e dados topográficos (ASTER GDEM) visando constatar a eficácia desta metodologia como ferramenta para mapeamentos geológicos regionais. A área de estudo situa-se a noroeste da carta topográfica Minas do Camaquã - RS, sendo que a anomalia estudada fica no centro da área de estudo.

\section{Abstract}

This paper uses as a main methodology the integration of aeromagnetic data on their transformations of your potential field, magnetic susceptibility data and topographical data (ASTER GDEM) in order to establish the effectiveness of this methodology as tool for regional geologic mapping. The study area is located northwest of the topographic map of the Minas Camaquã - RS, and the anomaly is studied in the center of the study area.

\section{Introdução}

A região abrangida pelo estudo que segue corresponde a uma área aproximada de $106 \mathrm{~km}^{2}$, que fica situada no município de Caçapava do Sul (RS) ao noroeste das "Minas do Camaquã", onde é comprovada a ocorrência de cobre além de ter existido lavra deste minério durante muitos anos.

A formação predominante da área é a Formação Guaritas; Fácie Varzinha ( $488 \mathrm{Ma})$ tendo como rochas predominantes arenitos e conglomerados, aflorando ao norte, sudeste, sudoeste e oeste da área de estudo, também se encontra na região outras formações que possuem menor importância para este trabalho, porém devem ser citadas; Formação Hilário, do Neoproterozóico ( 580Ma), caracterizado por conglomerados com clastos de rochas piroclásticas e vulcânicas; e pelitos. Basaltos da Formação Guaritas Membro Rodeio Velho ( 480Ma). Formação Santa Bárbara ( 520Ma); composta por pelitos e arenitos muito finos a médios. Rochas conglomeráticas olimíticas e polimíticas da Formação Santa Fé ( 540Ma). Depósitos aluvionares atuais, do Quaternário ( 65Ma).

A referência geológica deste trabalho toma como fonte de referência o mapa geológico da Folha Cachoeira do Sul
(CPRM, 2000), o qual é a carta geológica mais atual da região. Como será citada a seguir a região alvo pode se tratar de uma anomalia magnética proveniente de um corpo ainda não mapeado, sendo este trabalho uma contribuição a geologia regional.

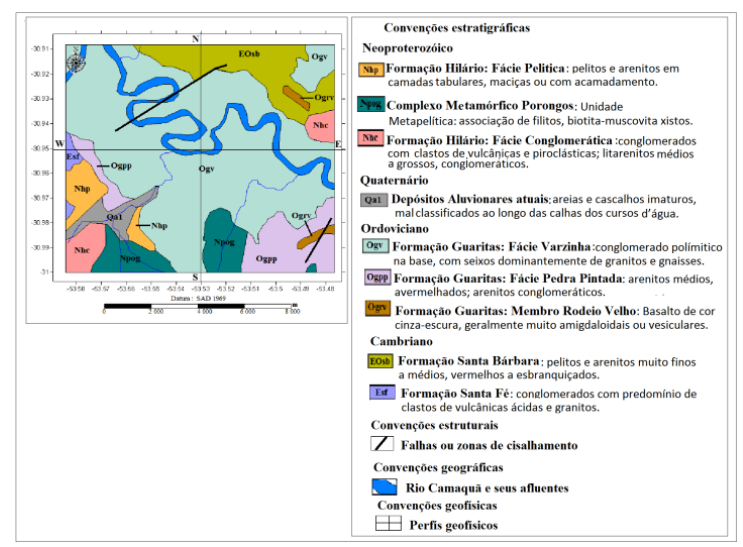

Figura 1 - Mapa geológico simplificado da região estudada, (modificado carta cachoeira 1: 250.000 CPRM, 2000).

\section{Metodologia}

Foi adotada como metodologia para este trabalho a integração de dados aeromagnéticos com dados de susceptibilidade magnética em conjunto com dados de altitude ortométrica. Inicialmente efetuou-se 0 processamento de dados Aeromagnéticos do levantamento aerogeofísico (CPRM, 1974), o campo potencial foi filtrado e transformado com diversas técnicas (Figura 2), ressaltando assim uma anomalia magnética ao centro da área de estudo, sendo esta anomalia magnética mais evidente no Filtro cosseno direcional, na segunda derivada vertical, na redução ao polo e no sinal analítico.

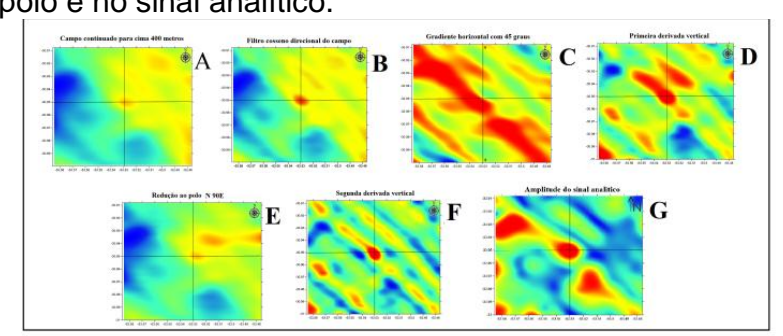

Figura 2: Filtros e transformações do campo potencial magnético efetuado no trabalho. A- Continuação para cima 400 metros. B- Filtro cosseno direcional. $C$ Gradiente horizontal com 45 graus. $D$ - Primeira derivada vertical. E- Redução ao polo. F- Segunda derivada vertical. G- Amplitude do sinal analítico. 
Para a confecção do mapa residual de anomalia magnética de intensidade total as técnicas de redução utilizadas, foram à remoção do IGRF, a filtragem cosseno direcional e a continuação para cima 400m.

Foram gerados conjuntamente com dados aeromagnéticos, mapas de altitude ortométrica subproduto da campanha ASTER GDEM (METI/NASA 2009) para a posterior verificação da topografia da região anômala.

Levantaram-se dados de susceptibilidade magnética visando verificar e complementar o comportamento magnético da região, e assim integrar com os dados do levantamento aeromagnético.

\section{Modelo Digital de Elevação do Terreno (DEM)}

No trabalho foram compilados dados de altitudes ortométricas para a área de estudo proveniente do ASTER GDEM (METI/NASA) estes produtos são um resultado de um consorcio entre a NASA e o Ministério da Economia, Comércio e Indústria do Japão (METI). Em Earth Resources Data Analysis Center (ERSDAC) e o United States Geological Survey (USGS) para construção de uma modelo digital de elevação global de livre acesso, os quais oferecem uma resolução espacial em longitude e latitude de aproximadamente 1 arco-segundo ( 30 metros) e uma resolução vertical de aproximadamente 7$14 \mathrm{~m}$ (DEM accuracy - stdev).

A Figura 3 mostra um mapa topográfico da área de estudo obtido a partir dos dados de altitudes ASTER GDEM. Posteriormente foi elaborado um Modelo Digital de Elevação do Terreno (DEM) conforme a Figura 3.

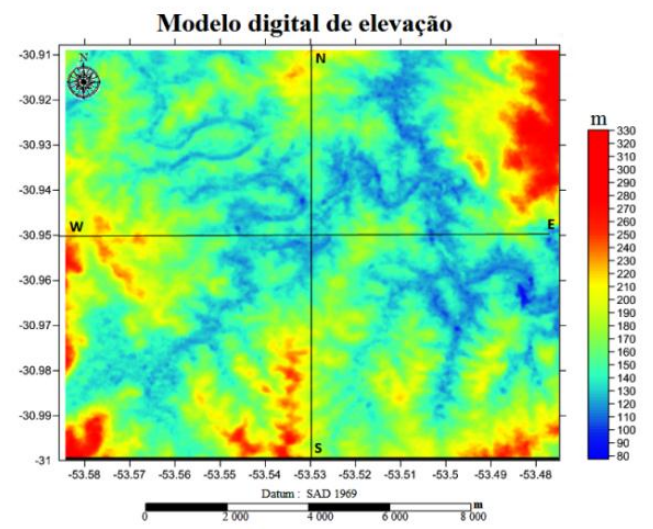

Figura 3 - Modelo Digital de Elevação do Terreno (DEM) baseados em dados ASTER.

Nota-se que nesta região existe predominantemente baixo relevo principalmente na porção central que é a região de interesse para o trabalho. A região com menor cota ortométrica está associada principalmente na região da Formação Guaritas; Fácie Varzinha ( 488 Ma).

\section{Filtro cosseno direcional.}

A utilização do filtro cosseno direcional, é necessária quando em um mapa interpolado os efeitos das linhas de vôo forem muito intensos, podendo interferir na interpretação dos resultados. A função do filtro cosseno é suavizar as feições que ocorrem em um determinado sentido, neste caso, das linhas de vôo. Uma característica da aplicação desse filtro é o valor usado no grau de suavização. Quanto mais baixo, mais abrupto é o valor do filtro, e o inverso ocasiona a suavização excessiva, apresentando ainda uma tendência a criar uma nova feição. O filtro utiliza a seguinte equação:

$$
L(E, N)=\left|\cos ^{n}\left(\alpha-\theta+\frac{\pi}{2}\right)\right|
$$

Onde "n" é o grau de suavização, $\alpha$ é a direção da feição e $\theta$ é a direção do filtro (MagPick V 3.05).

O filtro cosseno direcional (figura 4) foi aplicado nos dados brutos, justamente para reduzir as tendências causadas pelas linhas de vôo.

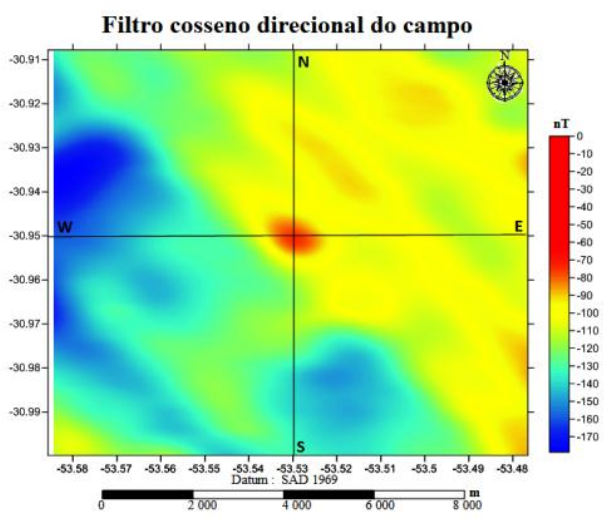

Figura 4 - Filtro cosseno direcional aplicado nos dados brutos.

\section{Continuação para cima 400 metros}

Depois de aplicado o filtro citado anteriormente foi aplicado à transformação do campo pela técnica da continuação para cima sobre o grid gerado pelo filtro cosseno direcional, gerando assim a grid final para se efetuar todas as posteriores transformações do campo (figura 5).

O filtro de continuação para cima tende a atenuar as anomalias de alta frequência, e manter aquelas anomalias de maior comprimento de onda, que podem estar associadas à presença de fontes magnéticas profundas. No domínio espectral a continuação para cima pode ser escrita como:

$$
F(u, v)=s(u, v) \times f(u, v) \Longrightarrow F(u, v)=\frac{s(u, v)}{1+\alpha s(u, v)^{2}} \times f(u, v)
$$

Sendo: $f(u, v)$ é o espectro do campo original a ser transformado; $\mathrm{F}(\mathrm{u}, \mathrm{v})$ é o espectro do campo transformado; $s(\mathrm{u}, \mathrm{v})$ é a componente espectral da transformação, e $\alpha$ o parâmetro de regularização $A$ (MagPick V 3.05). 


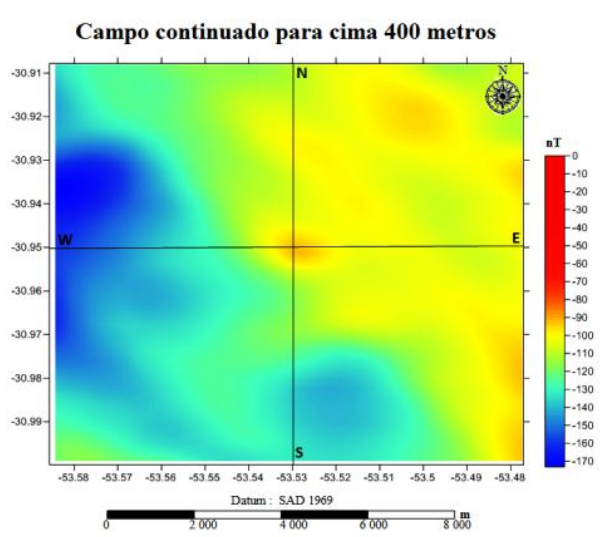

Figura 5- Mapa continuado para cima 400 metros

\section{Redução ao pólo}

O uso de um mapa de redução ao pólo consiste em fazer com que a anomalia magnética seja independente da direção do corpo litológico, já que esta é uma característica que os corpos apresentam quando estão situados em pólos magnéticos. Para que possa ser realizado este procedimento da transferência da anomalia, é necessário obter alguns dados físicos como a inclinação magnética, a declinação magnética e o campo total.

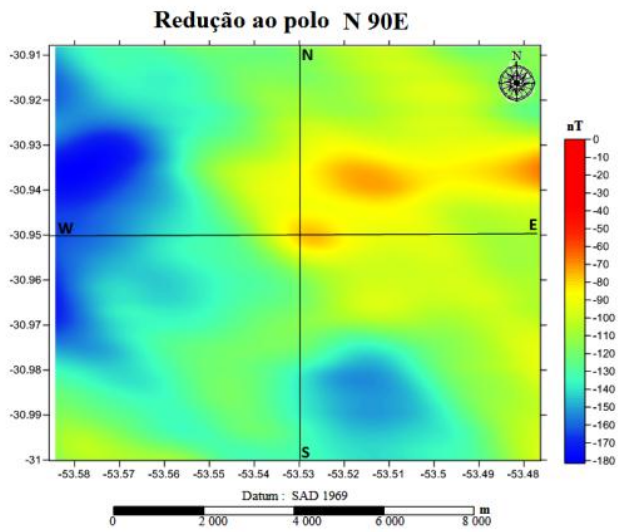

Figura 6 - Redução ao polo com a direção da linha do norte no sentido horário $90^{\circ}$

\section{Amplitude do Sinal Analítico (ASA).}

Nabighian $(1972,1984)$ desenvolveu o conceito do sinal analítico 2-D. Uma importante característica desta técnica é a independência que ela tem com relação à direção do vetor magnetização da fonte. A amplitude do sinal analítico é simplesmente relacionada à intensidade de magnetização.

Roest et al., (1992) mostraram que a amplitude (valor absoluto) do sinal analítico 3-D, em um ponto qualquer com coordenada $(x, y)$, pode ser facilmente obtido a partir de três gradientes ortogonais do campo magnético total usando a seguinte expressão:

$$
|\mathrm{A}(\mathrm{x}, \mathrm{y})|=\sqrt{\left(\frac{d T}{d x}\right)^{2}+\left(\frac{d T}{d y}\right)^{2}+\left(\frac{d T}{d z}\right)^{2}}
$$

Onde $|\mathrm{A}(\mathrm{x}, \mathrm{y})|$ é a amplitude do sinal analítico no ponto de coordenadas $(x, y)$. $T$ é a anomalia magnética de intensidade total no ponto de coordenadas $(x, y)$.

Uma vantagem fundamental que oferece o mapa de sinal analítico (figura 7), é que ele constitui uma boa ferramenta para selecionar anomalias de interesse geológico, já que seu algoritmo permite transformar as variações em diferentes direções, em variações somente positivas, que exibem um resultado que se corresponde diretamente com o grau de magnetização que apresentam as rochas.

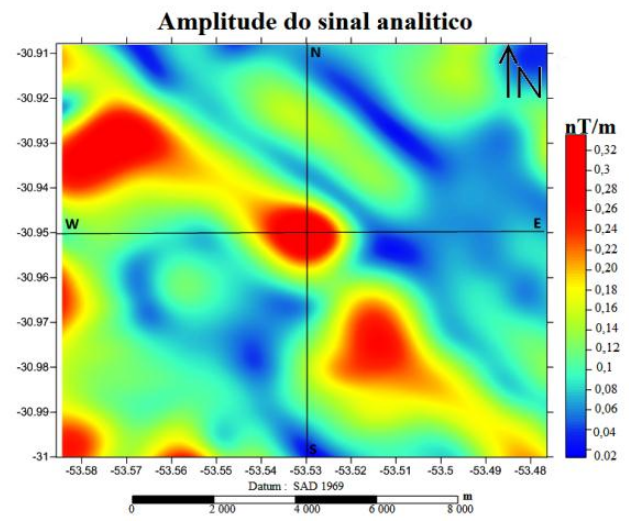

Figura 7- Mapa da amplitude do sinal analítico (ASA)

\section{Derivadas Verticais}

Obtêm-se a segunda derivada vertical da anomalia magnética de Intensidade total em um ponto, derivando a 1․ Derivada vertical, e têm-se a seguinte expressão:

$$
\begin{gathered}
f_{z}(x, y)=-\left.\frac{\partial \phi(x, y, h)}{\partial z}\right|_{z=0} \\
=\frac{1}{(2 \pi)^{2}} \iint_{-\pi}^{\pi} d \Phi_{0}(u, v) s \exp (j(u x+v y))
\end{gathered}
$$

A resposta do filtro para o cálculo da derivada vertical é dado por: (Naidu, P. S et al 1998).

$$
A(u, v)=\sqrt{u^{2}+v^{2}}
$$

Do mesmo modo, para o cálculo da segunda derivada vertical a resposta do desejada do filtro é dada por (Naidu, P. S et al 1998)::

$$
A^{2}(u, v)=u^{2}+v^{2}
$$

Para calcular as Derivadas Direcionais a partir de uma grid, as derivadas analíticas são aproximadas por fórmulas de diferenças finitas. As Derivadas Direcionais permitem ressaltar aquelas anomalias magnéticas de baixa intensidade em zonas onde a resposta magnética dos corpos intrusivos de composição básica possam ter seus efeitos atenuados pela presença de espessos pacotes de sedimentos situados em níveis mais rasos. 
Os produtos destas transformações encontram-se a seguir nas figuras 8 e 9 .

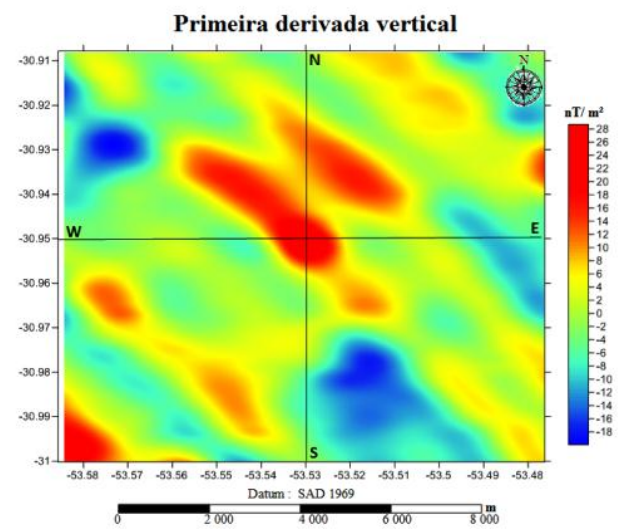

Figura 8: Primeira derivada vertical nota-se que esta transformação não conseguiu delimitar muito bem as bordas do corpo.

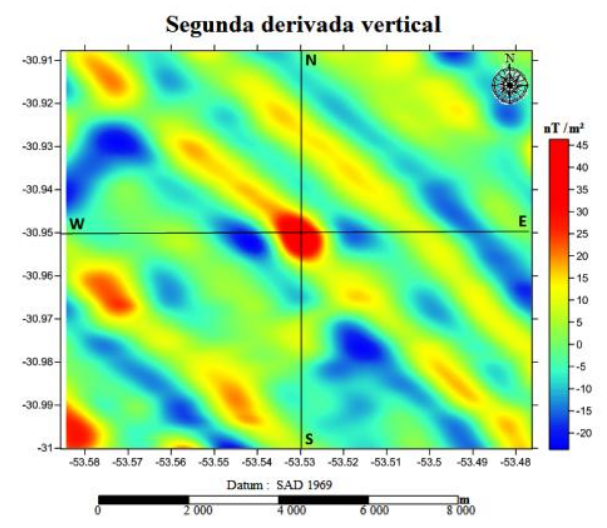

Figura 9: Segunda derivada vertical nota-se que esta transformação conseguiu delimitar de uma maneira eficiente às bordas da anomalia.

\section{Gradiente Horizontal}

O Gradiente horizontal do campo magnético de intensidade total (figura 10) foi obtido a partir de uma malha de valores de Anomalia Magnética de Intensidade Total, onde as derivadas analíticas são aproximadas por fórmulas de diferenças finitas. Essa técnica tem a característica de realçar através de máximos e mínimos, respectivamente, as bordas e os centros das fontes.

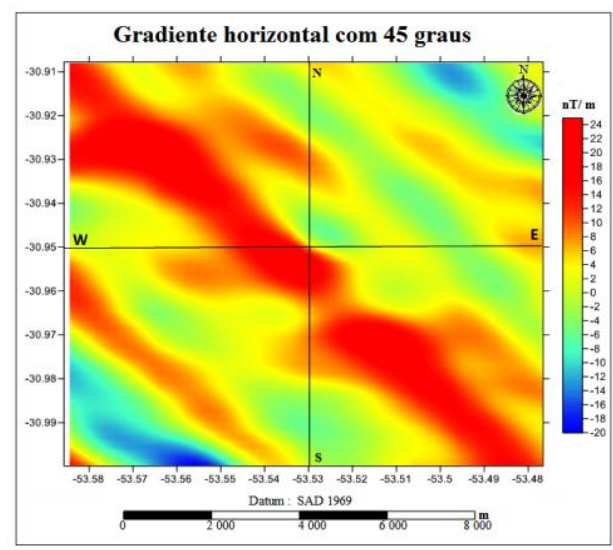

Figura 10: Gradiente Horizontal da região alvo. Nota-se que este filtro com esta direção mostrou-se ineficiente para a caracterização da feição geológica investigada.

\section{Susceptibilidade Magnética}

Para o levantamento de susceptibilidade magnética utilizou-se o aparelho KT. 10 Magnétic SusceptibilityMeter Terraplus fornecido pela Universidade Federal do Pampa.

As medidas foram efetuadas em diferentes amostras tentando tomar como direção preferencial os perfis geofísicos N-S e W-E (conforme figura 11), todas as medidas foram feitas em cima da Formação Guaritas Fácie Varzinha, que é predominantemente composta por arenitos, ouve um adensamento de pontos na região anômala, que foi tomada como base a região indicada pelo mapa do sinal analítico. No levantamento terrestre em cada localidade, realizaram-se diversas medições para gerar um melhor tratamento estatístico dos dados coletados. O valor final de susceptibilidade magnética de cada ponto encontra-se nas tabelas que seguem abaixo sendo que o valor representado é o valor médio das medições de cada amostra.

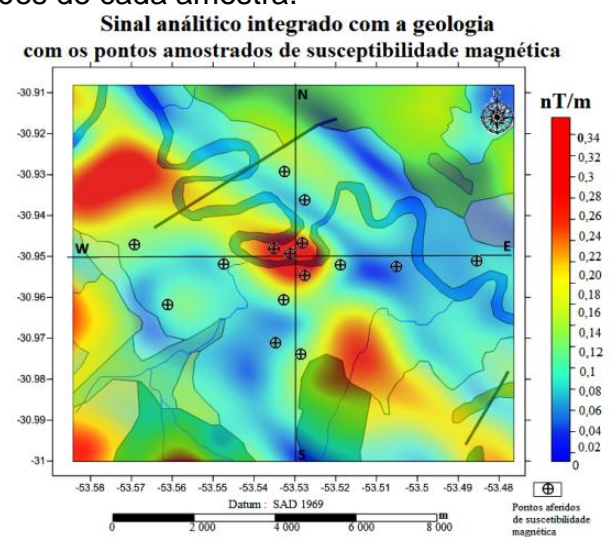

Figura 11: Mapa integrado da geologia com o sinal analítico e os pontos de medidas de susceptibilidade magnética. 
Tabelas dos perfis: Valores médios de susceptibilidade magnética (em negrito os valores correspondentes à região anômala ao centro da área de estudo).

\begin{tabular}{|c|c|c|c|c|c|c|c|}
\hline \multicolumn{2}{|c|}{ Perfil Oeste Leste } & \multirow{2}{*}{$\begin{array}{l}\text { Número de } \\
\text { medidas no ponto }\end{array}$} & \multirow{2}{*}{$\begin{array}{l}\text { K médio } \\
\left({ }^{*} 10^{\wedge}-3 \mathrm{SI}\right)\end{array}$} & \multicolumn{2}{|c|}{ Perfil Norte Sul } & \multirow{2}{*}{$\begin{array}{l}\text { Número de } \\
\text { medidas no ponto }\end{array}$} & \multirow{2}{*}{$\begin{array}{l}\text { K médio } \\
\left({ }^{*} 10^{\wedge}-3 \mathrm{SI}\right.\end{array}$} \\
\hline Long. & Lat. & & & Long. & Lat. & & \\
\hline$-53,5688$ & $-30,9463$ & 9 & 0,0277 & $-53,5321$ & $-30,9288$ & 5 & 0,0605 \\
\hline$-53,5608$ & $-30,9612$ & 6 & 0,0956 & $-53,5272$ & $-30,9354$ & 12 & 0,1155 \\
\hline$-53,5473$ & $-30,9516$ & 8 & 0,2514 & $-53,5276$ & $-30,9458$ & 15 & 19,5259 \\
\hline$-53,5349 \mid$ & $-30,9477$ & 15 & 23,1933 & $-53,5265$ & $-30,9542$ & 15 & 21,1919 \\
\hline$-53,5308$ & $-30,9487$ & 15 & 20,5258 & $-53,5321$ & $-30,9598$ & 9 & 0,0876 \\
\hline$-53,5184$ & $-30,9514$ & 4 & 0,0471 & $-53,5346$ & $-30,9706$ & 7 & 0,0112 \\
\hline$-53,5046$ & $-30,9520$ & 5 & 0,1253 & $-53,5279$ & $-30,9734$ & 9 & 0,0173 \\
\hline$-53,4851$ & $-30,9506$ & 10 & 0,0544 & & & & \\
\hline
\end{tabular}

\section{Resultados e Discussões}

Como produto final gerou-se um modelo digital de elevação integrado com uma "layer" do mapa do sinal analítico (figura 12), indicando assim que a região da anomalia de alta amplitude fica localizada em uma região de menor cota que as demais formações da área de estudo.

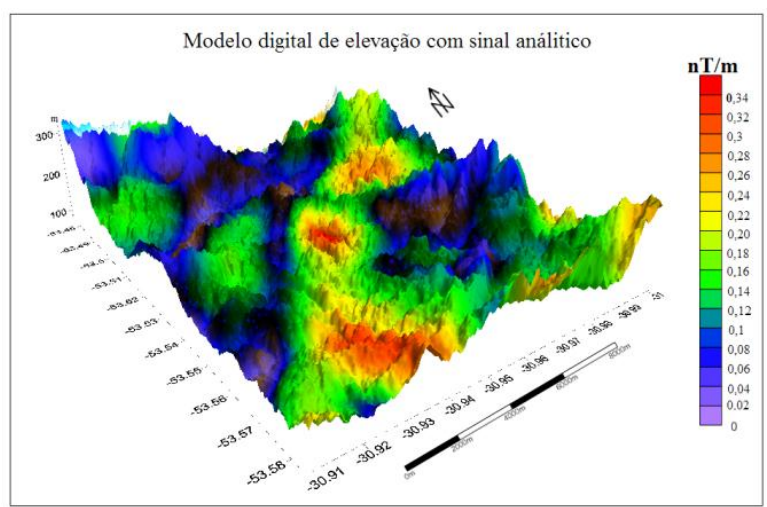

Figura 12: Modelo digital de elevação integrado com o sinal analítico.

Também como produto foi gerado diversos gráficos de cada perfil em cada transformação e filtro do campo potencial (Figura 13 e 14, ao final do trabalho). Foram plotados gráficos do comportamento da topografia da região dos perfis, podendo assim verificar o comportamento da Anomalia Magnética ao longo dos perfis W-E e N-S.

\section{Discussão e Conclusões}

A pesquisa demonstrou a eficiência de uma metodologia de processamento e integração de dados geofísicos conjuntamente com dados de modelo de elevação digital (DEM) podendo servir de ferramenta para o apoio e esclarecimento da cartografia geológica regional.

Conforme verificado nota-se um comportamento magnético anômalo ao centro da região estudada sendo que esta anomalia de maior intensidade situa-se em uma região de baixa altitude. Também se nota que no mapa geológico mais atual disponível da região, a anomalia fica inserida apenas em um contexto sedimentar uma região abrangida apenas pela Formação Guaritas; Fácie Varzinha ( 488 Ma).

A integração dos dados aeromagnéticos com os dados de susceptibilidade magnética apenas aumenta a suspeita de existência de um corpo, com altas propriedades magnéticas, ainda não mapeado na área.
As transformações que ressaltam as bordas dos corpos em 2D mostram que o provável corpo deve possuir uma aparência circular, extrapolando um pouco os dados uma feição similar a um "neck vulcânico". Porém, posteriormente serão efetuados levantamentos magnetométricos terrestres de detalhe ( 1: 25 000) na região, com o intuito de realizar a modelagem do possível corpo.

O trabalho faz parte de um projeto de pesquisa em andamento que tem como objetivo geral a integração e posterior interpretação de dados oriundos de Levantamentos Aerogeofísicos e Levantamentos geofísico terrestres com a finalidade de oferecer um subsídio para o mapeamento geológico regional e esclarecer questões relacionadas com a geometria da estruturação geológica da região da Bacia do Camaquã no estado do Rio Grande do Sul. Posteriormente serão efetuados levantamentos geofísicos de detalhe na região alvo com o intuito de se efetuar uma modelagem do corpo.

\section{Agradecimentos}

Agradeço a todos que me guiaram a ajudaram nessa caminhada gratificante desta linda profissão que é a geofísica. Como tio Frank Sherman Land disse um dia: "O Princípio que importa". Por isso agradeço a minha família, minha mãe Sandra, meu Pai Luis e meu Irmão Arthur. Agradeço a meus tios e irmãos do capitulo Paz e Prosperidade $\mathrm{n}$ 715. Agradeço especialmente ao meu orientador e grande amigo/geofísico Mario Rosales e toda minha equipe de trabalho que são pessoas que só me fazem crescer Cauê, Guilherme, Marcos, Lucas, Ana. Agradeço também aos meus colegas na CPRM, Andriotti e Ana Claudia. Não esquecendo também de meus colegas de estágio de férias da CDC engenharia os geofísicos Marcelo Ambrósio e André e aos técnicos de campo Adriano e Renato que tanto me ensinaram.

\section{Referências}

ASTER Global DEM Validation Summary Report from http://www.gdem.aster.ersdac.or.jp/index.jsp

CPRM - Serviço Geológico do Brasil. Geologia e Metalogênese. FOLHA SH. 22-Y-A, Estado do Rio Grande do Sul, Escala 1:250.000. Brasília: 2000. CDROM.

Jacson, V.N.; Ramos, V.A.; Terry, S.A. \& Zuzek, A.B. Projeto Aerogeofísico Camaquã, Estado do Rio Grande do Sul Porto Alegre, Texas Instruments: Convênio Departamento Nacional da Produção Mineral/Companhia de Pesquisa de Recursos Minerais/CENEN, 208 p., 1974.

GEOSOFT. 2005. Data Processing and Analysis System for Earth Science Applications. User Guide.

Grapher - Version 8.1.388. Graphing System. Copyright 1992 - 2009, Golden Software Inc. 
MagPick V 3.05 - Magnetic processing and interpretation. 1996 - 1998 Hamburg University, Germany. 2010 Geometrics, Inc. USA

Nabighian, M.N. 1972. The analytical signal of twodimensional magnetic bodies with poligonal cross-section its properties and use for automated anomaly interpretation. Geophysics, v. 37, p. 507-517.

Nabighian, M. N., 1984. Toward a three-dimensional automatic interpretation of potential field data via generalized Hilbert transforms: Fundamental relations: Geophysics,, 49, 780-786.

Oasis Montaj, V 6.4.2 (HJ) - Standart Edition. Copyright 2007, Geosoft Inc.

Naidu, P. S.; Mathew M. P., 1998. Analysis of Geophysical Potential Fields - A digital signal Processing Approach. Amsterdam. Elsevier, p. 176-177.

Roest W.R., Verhoef J., Pilkington M. 1992. Magnetic interpretation using the 3-D analytic signal. Geophysics 57, 116125.

Surfer, Version 9.11.947 - Surface Mapping System, Copyright 1993 - 2010Golden Software, Inc.

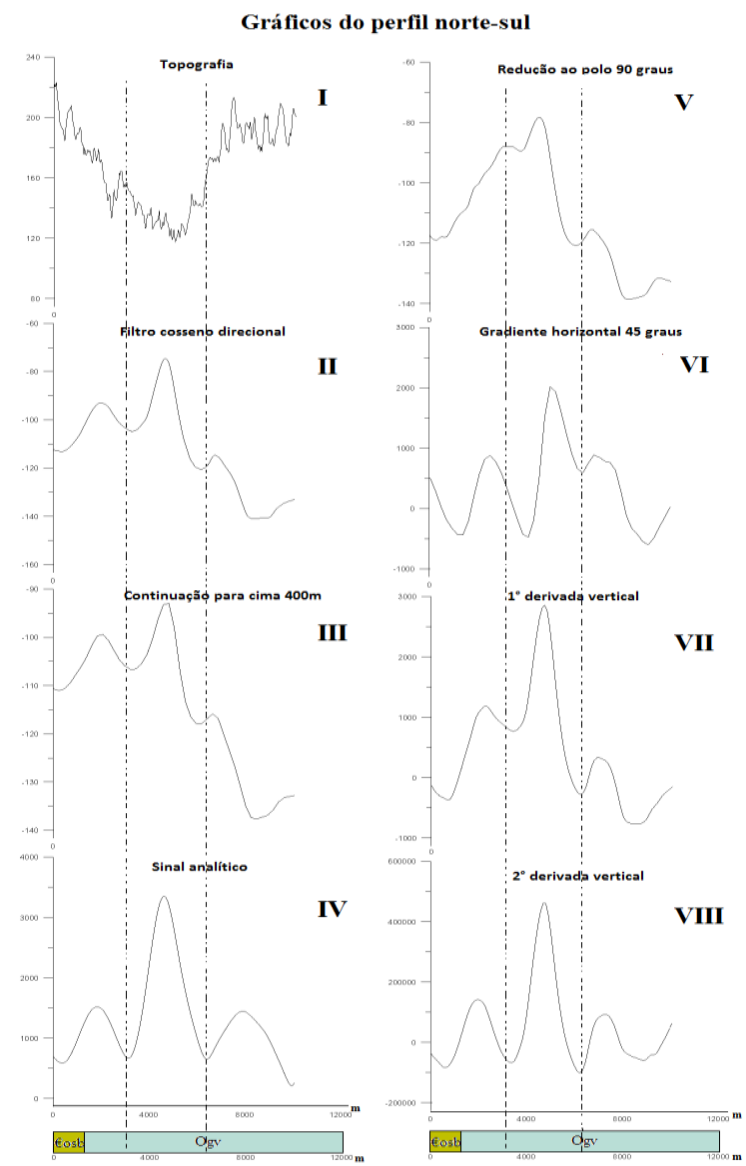

Figura: 13: Gráficos do perfil Norte-Sul.

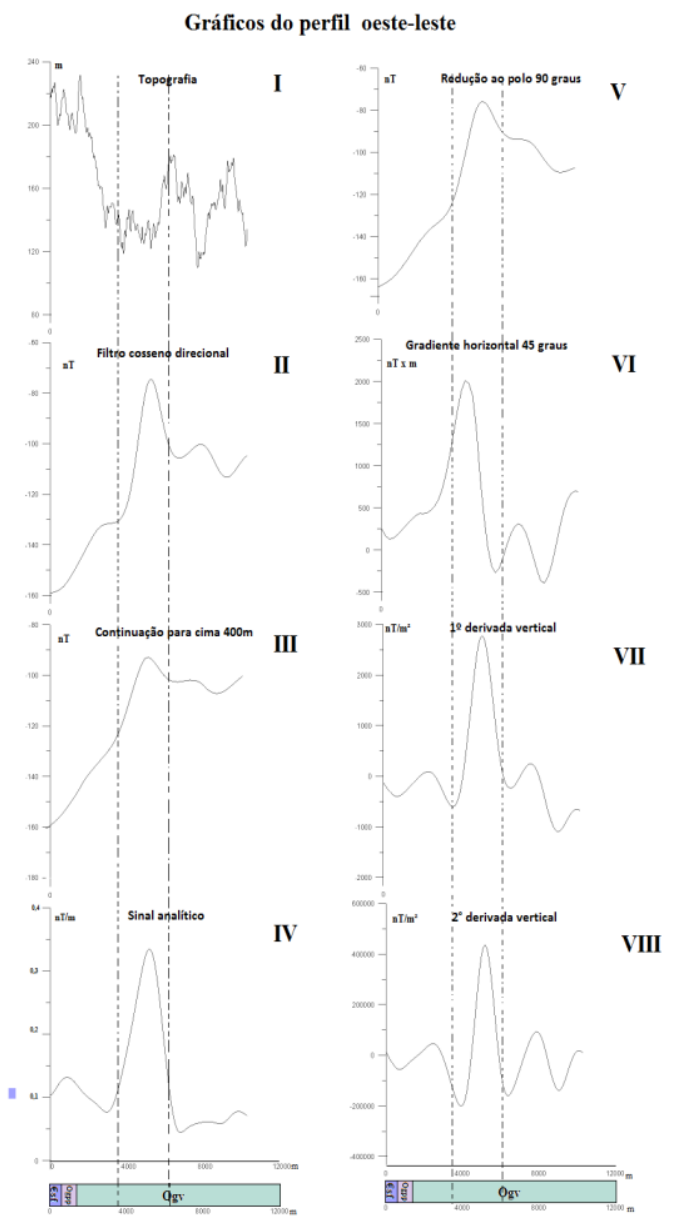

Figura 14: Gráficos do Perfil Oeste-leste.

Sendo que em ambas as integrações gráficas. - I topografia; II - Filtro cosseno direcional; -III- Continuação para cima 400 metros; -IV- Amplitude do Sinal Analítico; V- Redução ao polo; -VI- Gradiente Horizontal 45 graus; VII- Primeira derivada vertical; -VIII- Segunda derivada vertical. 